\title{
Conciencia lingüística en la construcción de discursos multilingües: la intercomprensión espontánea en portuñol
}

\author{
María Matesanz del Barrio (1) @ \\ Universidad Complutense de Madrid (UCM), España
}

Resumen. La producción de discursos multilingües es muy baja en relación a la generación de discursos monolingües, máxime si se tiene en cuenta la configuración multilingüe de nuestras sociedades. Los hablantes tienden a conformar discursos monolingües no solamente cuando se comunican en su lengua materna (L1), lo que es evidente, sino cuando la comunicación se realiza en una segunda lengua (L2). Localizar en la red producciones orales y escritas multilingües no resulta tarea fácil. La preferencia por la comunicación monolingüe en segundas lenguas responde a diversos factores, entre los que destacan la eficiencia comunicativa y el prestigio de la comunicación monolingüe, aspecto este último de gran influencia sobre los hablantes. En situaciones comunicativas formales en L2 no hay duda de que el discurso que domina es monolingüe. También en las comunicaciones no formales, pero en entornos naturales distendidos se observa una mayor libertad en la elección discursiva y la conciencia lingüística de los hablantes influye positivamente en la generación de discursos multilingües. Nos acercaremos a esta cuestión desde la perspectiva de la intercomprensión espontánea en español y portugués, partiendo de un corpus de estudio en el que se han registrado producciones orales y escritas de intercomprensión espontánea. Su análisis nos ha permitido observar una conciencia lingüística favorable a la interacción en distintas lenguas, lo que da entrada de forma natural al discurso multilingüe.

Palabras clave: discurso multilingüe; conciencia lingüística; intercomprensión; portuñol.

\section{A consciência linguística na construção de discursos multilíngues: a intercompreensão espon- tânea em portunhol}

Resumo. A produção de discursos multilíngues é escassa em relação à geração de discursos monolíngues, especialmente se a configuração multilíngue de nossas sociedades é levada em conta. Os falantes tendem a conformar discursos monolíngues não apenas quando se comunicam em sua língua materna (L1), o que é evidente, mas também quando a comunicação ocorre em uma segunda língua (L2). Encontrar na internet produções orais e escritas multilíngues não é uma tarefa fácil. A preferência pela comunicação monolíngue em segundas línguas responde a vários fatores, entre os quais se destacam a eficiência comunicativa e o prestígio da comunicação monolíngue, sendo este último aspecto de grande influência nos falantes. Em situações comunicativas formais em L2 não há dúvida de que o discurso dominante é monolíngue. Também em comunicações não formais, mas em ambientes naturais observa-se maior liberdade na escolha discursiva e a consciência linguística dos falantes influencia positivamente o surgimento de discursos multilíngues. Abordamos esta questão da perspectiva da intercompreensão espontânea em espanhol e português a partir de um corpus de estudo no qual foram registradas produções orais e escritas de intercompreensão espontânea. Sua análise permitiu-nos observar uma consciência linguística favorável à interação em diferentes línguas, o que possibilita de forma natural o discurso multilíngue.

Palavras-chave: discurso multilíngue, consciência linguística, intercompreensão, portunhol

\section{Linguistic awareness in the construction of multilingual discourses: spontaneous intercompre- hension in Portuñol}

Abstract. The production of multilingual discourses is very low if the current multilingual configuration of our societies is considered. Speakers tend to generate monolingual discourses, not only when they communicate in their mother tongue, which is obvious, but when communication is performed in a second language. The realisation of this fact is easily seen on the Internet, since locating oral and multilingual written productions on the network is not an easy task. The preference for monolingual communication in second languages responds to a number of factors, including the communicative efficiency and the prestige of monolingual communication, an aspect of great influence on speakers. In formal communicative situations there is no doubt that the discourse that dominates is monolingual. Also in non-formal communications but in natural environments there is greater freedom in the discursive choice and linguistic awareness of speakers has a positive influence on the construction of multilingual discourses. We approach this issue from the perspective of spontaneous intercomprehension in Spanish and Portuguese based on a corpus of study in which 
oral and written productions of spontaneous intercomprehension have been recorded. The analysis has allowed us to observe a linguistic awareness favourable to interactions in different languages which naturally enters multilingual discourse.

Keywords: multilingual discourse, language awareness, intercomprehension, portuñol.

\section{Introducción}

La comunicación entre hablantes de distintas lenguas maternas genera, preferentemente, discursos monolingües en una segunda lengua compartida por ellos. Es evidente en entornos formales de comunicación, aunque los hablantes se conducen con mayor libertad cuando se encuentran en otro tipo de situaciones discursivas. Los hablantes no siempre optan por el discurso monolingüe y generar discursos multilingües, entre ellos, discursos bilingües. La elección de la lengua o lenguas que eligen los hablantes en una interacción comunicativa en la que alguno de ellos no es nativo es variable y responde, principalmente, al peso que tiene dos factores lingüísticos: la competencia plurilingüe (Coste, Moore \& Zarate, 2009) y la acomodación lingüística (Street \& Giles, 1982; Beebe \& Giles, 1984; Giles, 2016). También hay que tener en cuenta otros factores, como las creencias lingüísticas, que han sido estudiadas en contextos de aprendizaje (Horwitz, 1988, 1999; Riley, 1997; Jee, 2017), pero más difíciles de precisar en contextos naturales.

Los entornos naturales de comunicación conceden mayor libertad a los hablantes y las soluciones discursivas que adoptan son, en consecuencia, más variadas. También lo son, los factores que influyen en las decisiones discursivas, ya que las interacciones carecen del control que en mayor o menor medida se produce, por ejemplo, en el aula o en interacciones profesiones (Matesanz del Barrio, 2015). En relación con las creencias, se puede situar la conciencia lingüística ya que las ideas de los hablantes sobre las lenguas en general o sobre lenguas concretas influyen en la toma de decisiones al establecer una comunicación. Una de estas decisiones puede ser la creación de discursos multilingües, entre los que consideramos la intercomprensión. En concreto, nos centramos en este estudio en un tipo de discurso bilingüe espontáneo bien identificado por los hablantes, el portuñol. Planteamos, en primer lugar, cómo la conciencia lingüística puede actuar positivamente en discursos espontáneos y cómo puede influir en la construcción discursiva multilingüe. Nos centramos en los discursos multilingües que genera la intercomprensión, en particular en la intercomprensión bilingüe en español y portugués y la generación de un portuñol no estable. Para poder analizar estas producciones hemos realizado un pequeño estudio experimental, para el que hemos elaborado un corpus de interacciones multilingües orales y escritas con base en el español y portugués, obtenido de internet. La observación y análisis de las muestras de ese corpus y de un subcorpus monolingües con 
reflexiones metalingüísticas de los hablantes sobre las interacciones españolportugués y portuñol nos ha permitido comprender cómo actúan los hablantes y qué les mueve a generar este tipo de discurso multilingüe.

\section{La conciencia lingüística en la construcción discursiva}

La conciencia lingüística de los hablantes se ha tratado desde la perspectiva del conocimiento lingüístico y metalingüístico de los aprendientes, inicialmente por parte de nativos en el proceso de adquisición de su lengua (Karmiloff-Smith, 1975; Pinto, 1999). Posteriormente se iniciaron estos trabajos en hablantes de una segunda lengua (L2) con Hawkins (1984) y en hablantes de una tercera lengua, en definitiva, hablantes multilingües (Jessner, 2005, 2006). De hecho, Jessner (2006) presenta un interesante estado de la cuestión sobre la conciencia lingüística y lo utiliza en su estudio como un término que incluye, por un lado, la conciencia lingüística y la conciencia sobre la lengua; y, por otro, como sinónimo de conciencia metalingüística en hablantes multilingües (Jessner, 2006: 43). El trabajo de Jessner se centra en hablantes multilingües que tienen el inglés como tercera lengua y, además, su estudio se refiere principalmente a situaciones de aprendizaje formal. Sitúa su estudio en el marco de la enseñanza-aprendizaje, la línea de investigación que se ha seguido en las investigaciones sobre la conciencia lingüística en segundas lenguas. Además, hay que tener en cuenta las aportaciones de los trabajos que se centran más específicamente en el conocimiento lingüístico, muy relacionado con la conciencia lingüística, hasta el punto de que en ocasiones se han considerado sinónimos (Ellis, 2002, 2004, 2005).

La conciencia lingüística en segundas lenguas se ha analizado tempranamente desde la perspectiva educativa y curricular (Carrel, 1989; Garret \& James, 1992; James, 1999) y cuenta con un amplio desarrollo en todos los niveles lingüísticos (Denham \& Lobeck, 2010; Kennedy, 2012). Es difícil saber cómo interviene la conciencia lingüística en las decisiones que toman los hablantes en situaciones discursivas de contexto natural (no controladas) en relación a la elección del modelo discursivo (monolingüe o multilingüe) que van a utilizar para comunicarse. Los hablantes generan dos tipos de discursos, monolingües o multilingües, con variaciones que dan lugar a 8 modelos discursivos, algunos de ellos con variantes mínimas (Matesanz del Barrio, 2019). La elección del modelo discursivo que se va a generar es una decisión que de forma consciente o inconsciente toman los hablantes antes de iniciar una interacción comunicativa. No hay duda de que el modelo discursivo que eligen los hablantes nativos (HN) en toda interacción en la que previamente se han reconocido nativos es el discurso monolingüe en lengua materna. En este caso podemos considerar que no hay una reflexión 
lingüística previa, siempre que el hablante identifique a su interlocutor como nativo. El discurso monolingüe en lengua materna es completamente espontáneo entre aquellos hablantes que son nativos o bilingües ${ }^{1}$. Pero no siempre estos discursos monolingües entre $\mathrm{HN}$ se generan en la lengua materna de los hablantes ya que en contextos de aprendizaje de una L2 en el aula o en otras circunstancias de aprendizaje, como es el deseo de practicar una lengua entre nativos, el discurso se genera en una segunda lengua ${ }^{2}$. También encontramos un discurso monolingüe en $\mathrm{L} 2$, cuando los hablantes no quieren ser reconocidos como no nativos, o bien quieren mostrar su integración en aquellos casos en los que los hablantes son extranjeros. Estos dos últimos casos de nativos que se comunican generando un discurso en lengua no materna exigen, por parte de los hablantes, una reflexión sobre la lengua que van a usar, lo que indica una conciencia lingüística o toma de conciencia sobre las lenguas. No entraremos en esta fina diferenciación terminológica entre conciencia y toma de conciencia, porque, como señala la propia Jessner (2006: 41) establecer una distinción entre ambos conceptos es casi imposible. Pinto (1999, 2015) utiliza en italiano la palabra consapevolezza, término con el que indica que el individuo realiza una reflexión personal sobre el objeto y toma conciencia de él, en este caso la lengua.

Las interacciones de hablantes nativos con no nativos (HN/HNN) y de hablantes no nativos entre ellos (HNN/HNN) presentan, inicialmente, una mayor complejidad en cuanto a la lengua o lenguas en las que va a desarrollarse el discurso, bien sea monolingüe o bilingüe. Los discursos monolingües en L2 generados por grupos de hablantes en los que no se comparte una lengua materna requieren, al menos, una evaluación previa de la lengua elegida para la comunicación. El caso más paradigmático de esta situación discursiva es la generación de discursos monolingües en una lengua franca, como puede ser el inglés o bien cualquier lengua de difusión, las consideradas lenguas internacionales. El recurso a una lengua franca entre hablantes nativos y no nativos requiere, además, un grado de competencia en la L2 elegida suficiente para que la comunicación sea efectiva y pueda tener éxito comunicativo. Una competencia lingüística deficiente obliga, por ejemplo, a invertir más tiempo en la producción y también en la comprensión del mensaje, lo que supone una ralentización de la comunicación (Matesanz del Barrio, 2015, p. 170), algo que no siempre es bien aceptado por los interlocutores.

${ }^{1}$ De modo general, al hablar de nativos en este trabajo se está incluyendo a hablantes bilingües en el sentido de Bialystok (2001: 4-5) o bilingües equilibrados, según la denominación de Etxeberria (1995).

${ }^{2}$ En este estudio, salvo que se indique lo contrario, por segunda lengua (L2), entendemos cualquier lengua no materna que utilicen los hablantes para generar un discurso, independientemente del orden de adquisición que ocupe esa lengua en el conocimiento lingüístico del hablante. 
La valoración de la comunicación en L2 requiere, sin duda, una reflexión sobre la capacidad lingüística propia, además de la evaluación de las competencias lingüísticas del interlocutor o interlocutores con quienes se quiere establecer una comunicación, como hemos señalado. Una posibilidad de éxito baja en la comunicación empuja a los hablantes a valorar otras opciones discursivas, entre ellas la generación de un discurso multilingüe. Es necesario señalar que los discursos multilingües pueden producirse entre hablantes plurilingües completamente competentes en la lengua o lenguas de su interlocutor, pero que por razones muy diversas, como pueden ser los hábitos lingüísticos que se establecen entre los interlocutores, utilizan más de una lengua en su discurso. Estas lenguas pueden ser sus propias lenguas maternas o una $\mathrm{L} 2$, pero la opción que realizan hace que generen un discurso multilingüe. Un ejemplo común de este discurso multilingüe es el discurso que construyen los niños bilingües/trilingües hablando con sus padres, con quienes comparten lenguas, pero la diferencia está en que para los padres una de esas lenguas es la lengua materna y la otra u otras son L2.

Los discursos multilingües se generan con frecuencia en situaciones de lenguas en contacto ${ }^{3}$. Los cambios de código ${ }^{4}$, vistos desde el bilingüismo de lenguas en contacto con hablantes competentes, fueron observados y estudiados por primera vez por Weinreich (1953) en conversaciones entre hablantes suizos con distintas lenguas maternas. La producción de discursos multilingües no se limita a situaciones de lenguas en contacto y encontramos muestras de discursos multilingües en interacciones de aprendientes de L2 (Alvanoudi, 2018; Cheng, 2013; Dewaele, 1998, 2001; Sukwon \& Yang-soo, 2015). Sin embargo, no disponemos de estudios sobre los cambios de código espontáneos en hablantes no bilingües que no están en situación de lenguas en contacto ${ }^{5}$, aspecto que nos interesa particularmente en este estudio. Los hablantes también producen discursos multilingües cuando tiene una baja competencia lingüística y buscan lograr una comunicación aceptable. En estos casos de producción de discurso multilingüe nos encontramos con dos situaciones distintas: por un lado, (i) los hablantes comparten lenguas, pero tienen una competencia débil en ellas; y, por otro, (ii) los hablantes no comparten lenguas, ni maternas ni segundas lenguas, pero alguna de las lenguas que poseen los hablantes ( $L 1-L 2, L n)$ tiene relación tipológica, es decir, alguna de sus lenguas pertenecen a la misma familia lingüística. En el primer caso (i) nos encontramos con producciones discursivas multilin-

\footnotetext{
${ }^{3}$ Para una revisión actualizada del multilingüismo y lenguas en contacto, véase Moreno Cabrera (2016).

${ }^{4}$ Entendemos cambios de código de forma no restrictiva. Para una revisión bastante reciente de la cuestión puede consultarse Gardner-Chloros (2009).

${ }^{5}$ El estudio de Torres Cacoullos \& Travis (2016) aunque trata conversación espontánea; sin embargo, utiliza un corpus de hablantes bilingües en situación de lenguas en contacto.
} 
gües en las que pueden producirse alternancia de código, mezcla de códigos y discurso fusionado (Auer, 1998) y en el segundo caso (ii) estamos ante situaciones discursivas de intercomprensión (Matesanz del Barrio, 2019). Por supuesto, ambos casos pueden presentarse de forma combinada, como veremos en la parte experimental.

Aunque nos hemos detenido más en los discursos multilingües, dada su complejidad ${ }^{6}$, la construcción de discursos monolingües es la opción más utilizada por los hablantes cuando se producen interacciones entre HN-HNN, entre otras cosas, por su mayor eficiencia comunicativa. Posiblemente los dos elementos principales que más influyen a la hora de preferir discursos monolingües son la concisión del mensaje y la rapidez en su producción. Como puede apreciarse son condicionantes de tipo muy distinto, pero que resultan decisivas en la elección que hacen los hablantes. Pensemos en un intercambio comunicativo multilingüe en el que no todos los interlocutores tienen el mismo grado de competencia en las lenguas utilizadas en él. Esta circunstancia hace que la precisión en la formulación y/o en la comprensión pueda hacer perder efectividad al mensaje. Al mismo tiempo, una producción deficiente requiere más tiempo en la emisión y en la recepción del mensaje, lo que supone un inconveniente para la comprensión por parte de los interlocutores y también para alcanzar un desarrollo discursivo fluido y eficiente.

Además, no debemos olvidar que los discursos monolingües en L2 tienen una mayor aceptación entre los hablantes que los discursos multilingües, puesto que presuponen que lo hablantes tienen una elevada competencia lingüística en L2. La competencia lingüística en L2 es un valor que socialmente tiene una alta consideración y se relaciona con un mayor grado de formación académica, como consideran Gardner-Chloros, McEntee-Atalianis \& Finnis (2005) en relación a los cambios de código. La mayor o menor aceptación de un modelo discursivo determinado -monolingüe o bilingüe- responde a la consideración positiva o negativa que los hablantes tienen sobre ellos. Los factores que influyen en esta valoración son muy diversos, algunos de índole personales y, por tanto, de difícil tipificación, pero otros son más evidentes, como es la consideración general sobre el valor positivo de las lenguas. Una muestra de esta valoración positiva es el alto porcentaje de europeos que considera positivamente el aprendizaje de una lengua para sus hijos (Eurobarómetro 386) ${ }^{7}$. El valor que se le concede al conocimiento de lenguas es una muestra de conciencia lingüística. Esta conciencia permite a los hablantes emitir juicios sobre su conocimiento y competencia en ellas.

6 No obstante, no nos hemos referido a todas la posibilidades de discursos multilingües (Matesanz del Barrio, 2019) ya que no todos nos interesan para este estudio.

7 El 98\% de los entrevistados europeos consideró que aprender lenguas era bueno para sus hijos. 


\section{Intercomprensión espontánea español-portugués: el portuñol}

La intercomprensión (IC) habitualmente se define partiendo de la perspectiva del hablante de modo que, de forma simplificada, la intercomprensión consistiría en una estrategia comunicativa en virtud de la cual cada persona puede expresarse en su lengua y comprender la lengua del otro (Beacco et al. 2012; Degache \& Melo, 2008; Doyé 2005; Escudé \& Janin 2010; Beacco et al. 2015). La aproximación a la intercomprensión se ha realizado inicialmente desde una perspectiva de la didáctica de las lenguas, iniciada ya en los años setenta del siglo pasado en Francia (Dabène, 1975), pero que adquiere fuerza en la última década del siglo con la conformación de grupos de investigación en torno al liderazgo de L. Dabène (Billiez, Degache \& Simon, 2013) y C. Blanche-Benveniste (Roubaud, 2010) en proyectos de dimensión europea, a los que hay que añadir otros proyectos posteriores en estas iniciativas (Escudé \& Janin, 2010; Di Gesù, 2017). La intercomprensión entendida como una metodología de enseñanza y aprendizaje de lenguas basada en la capacidad de los hablantes de comprender otras lenguas, utilizando para ello las similitudes con una o más lenguas de los hablantes (Beacco et al. 2015: 68), es uno de los campos fundamentales de estudio de la intercomprensión. La introducción de los enfoques plurales en la enseñanza de lenguas (Candelier, 2008), en la base del CARAP (Candelier et al. 2007) y sus aplicaciones curriculares para introducir el plurilingüismo y la interculturalidad en el ámbito escolar (Beacco et al. 2015) ha dado un nuevo impulso a la intercomprensión (DGLDFL, 2016; Hidalgo, 2015; Spita et al. 2016).

Pero podemos adoptar un punto de vista distinto para definir la intercomprensión, centrándonos en el discurso y no en el hablante. Desde esta perspectiva, la intercomprensión se entiende como un modelo de interacción comunicativa que genera un discurso multilingüe producido por locutores que no comparten las mismas lenguas ( $L 1, L 2)$ o que tienen una baja competencia lingüística en una segunda lengua común, lo que no les permite un intercambio comunicativo que genere un discurso monolingüe. En estos casos, los hablantes recurren para comunicarse a lenguas de su repertorio lingüístico que son de la misma tipología lingüística -lenguas maternas o segundas lenguas-, aunque es más frecuente la intercomprensión basada en el uso de las lenguas maternas de los interlocutores. La intercomprensión así entendida se caracteriza por la confluencia de tres elementos: (i) generación de un discurso multilingüe; (ii) no compartir lenguas o tener baja competencia en las lenguas que se comparten o; (iii) las lenguas utilizadas en la comunicación tienen que pertenecer a la misma familia lingüística, de modo que quede garantizado un nivel mínimo de comprensibilidad de los mensajes (Matesanz del Barrio, 2017, p. 134). 
La intercomprensión vista desde la perspectiva discursiva nos permite integrarla como modelos de interacción comunicativa en un marco más general y comprehensivo de la comunicación lingüística.

Los estudios experimentales de intercomprensión se han realizado, fundamentalmente, sobre interacciones producidas en entornos de enseñanza o de formación en intercomprensión. Estos marcos son controlados, en el sentido de que los hablantes tenían tareas asignadas ${ }^{8}$ pero, por supuesto, las interacciones de $\mathrm{IC}$ registradas pueden considerarse plenamente naturales. En este estudio nos interesa analizar producciones espontáneas de interacciones de IC en entornos no formales ya que queremos observar la conciencia lingüística de los hablantes en la producción de un modelo discursivo multilingüe en el que los hablantes no comparten lenguas. Nuestro interés se centra en conocer cómo y en qué contextos los hablantes recurren a esta modalidad de interacción. Para ello hemos optado por observar interacciones en español y portugués. El uso de portuñol no estable en las interacciones observadas introduce un elemento particularmente interesante de este discurso multilingüe producido de forma espontánea.

La palabra portuñol ${ }^{9} /$ portunhol (en español y portugués, respectivamente) es polisémica y hace referencia a distintas realidades lingüísticas. Al hablar de portuñol es necesario distinguir: (i) si nos estamos refiriendo a una de las variantes dialectales que tienen como base el español y el portugués y que se producen por un contacto entre las lenguas; o, (ii) si nos referimos a interacciones discursivas entre hablantes de español y portugués en las que se producen transferencia desde la lengua materna, cambios de código y otros fenómenos propios de la adquisición de una segunda lengua (Lipski, 2006; Corbella \& Fajardo, 2017), lo que en este estudio hemos denominado portuñol no estable. Este último caso puede responder a una situación de lenguas en contacto como en (i), pero no siempre es así. El portuñol como resultado de lenguas en contacto se localiza en un amplio y discontinuo espacio, ya que encontramos muestras de esta variante en algunas zonas de la frontera entre España y Portugal (Navas, 1998, 2015; Álvarez, 2017) y en la frontera entre Brasil y los países hispanohablantes con los que limita (Elizaincín, 1992, 2004; Moreno, 2001). Algunas de las variantes más estables del portuñol reciben denominaciones específicas, como, por ejemplo, en la Península Ibérica el barranqueño (Leite de Vaconcelos, 1955; Navas, 2011, 2014). En

${ }^{8}$ Algunas de las plataformas desarrolladas en los proyectos de IC enfocados a la formación permitían a los hablantes interaccionar entre ellos interaccionar entre ellos, como puede verse en uno de la descripción que de los proyectos Galanet y Galapro realiza Castro (2015).

${ }_{9}^{9}$ Aunque no es relevante en sí, resulta significativo que en la última edición del DLE no se haya registrado, lo que supone la baja incidencia de uso entre los hablantes. 
la frontera de Brasil con países hispanohablantes los dialectos más estables son el fronterizo, que se habla entre Brasil y Uruguay (Lipski 2009, Waltermire 2012) y el denominado portuñol salvaje, localizado entre las fronteras de Brasil, Paraguay y Argentina, en el que confluyen, además del portugués y el español, el guaraní y/o el inglés (Locane, 2015; Pires, 2017). Aunque se reconocen otras variantes, estas son de menor estabilidad y disponemos de una información bastante limitada de las relaciones interlingüísticas e interculturales de las lenguas implicadas (Campos, 2008; Lipski, 2017; Rojas, 2008; Sturza \& Tatsch 2016). Las variantes menos estables tienden a ser consideradas de menor prestigio por los hablantes (Sturza 2004, 2005). En cuanto al portuñol, tal y como lo presentamos en el punto (ii), apenas tenemos información de cómo se desarrolla, en qué circunstancias discursivas se utiliza y cómo lo ven los hablantes en relación a su lengua materna y con respecto a su repertorio lingüístico.

\section{Métodos y materiales}

Para el presente estudio cualitativo hemos elaborado un pequeño corpus de estudio con textos ${ }^{10}$ extraídos de internet en $2019^{11}$, tanto orales como escritos. Los textos espontáneos ${ }^{12}$ multilingües que registran interacciones de intercomprensión en dos o más lenguas son difíciles de detectar. En primer lugar, porque no se presentan como tales y los buscadores no permiten búsquedas combinadas de lenguas por lo que, a priori, es difícil saber qué lenguas van a aparecer en los textos, salvo la determinada en el buscador. Su localización es complicada porque la producción dominante en internet es claramente monolingüe y son pocos, relativamente, los textos multilingües en la red. Para la obtención de la muestra con la que trabajamos hemos utilizado palabras clave y sintagmas construidos sobre ellas. Las palabras clave ${ }^{13}$ básicas en ambas lenguas con las que hemos obtenido resultados positivos han sido: portugués, español, portuñol, conversación, diálogo, comentario, foro; y también itañol y frañol, estas últimas para ver si

10 Utilizamos texto en el sentido de cualquier producción lingüística, oral o escrita, realizada en cualquier formato, tradicional o multimedia. Un texto, en este sentido es, por ejemplo, un texto escrito de forma tradicional, un texto digital, una página web o un vídeo.

11 Las producciones tienen fechas variables y, además, no siempre tenemos datos de la generación del texto. De los textos que hemos reunido, y tienen fecha, el más antiguo es de 2003.

12 Por textos espontáneos entendemos textos que se producen de forma natural, sin una preparación previa; por ejemplo, en las interacciones. Esto hace que descartemos producciones textuales que se ajustan a un guion de producción, en las que, por ejemplo, los diálogos están escritos previamente, aunque simulen naturalidad.

13 Todas las palabras clave se han utilizado en español y en portugués; por ejemplo, hemos utilizado separadamente portuñol y portunhol. 
los fenómenos eran similares, pero no incluimos ejemplos obtenidos con ellas en este trabajo. Las producciones textuales en internet en las que se habla sobre el portugués y el español conjuntamente son casi exclusivamente textos escritos monolingües. Las producciones orales en las que confluyen a la vez el español y el portugués son pocas y son vídeos enmarcados en la enseñanza de lenguas, directa o indirectamente. En particular, son verdaderamente escasas las interacciones espontáneas orales de intercomprensión en la red. En el caso que tratamos, interacciones en las que, al menos, se utilicen el español y el portugués de forma natural hemos localizado algunos ejemplos, aunque también estos textos tienen una finalidad lingüística.

Además de las interacciones, hemos recopilado también una muestra de textos monolingües en los que se habla sobre el portuñol, en tanto que variante no estable de las interacciones de hablantes de portugués y español. Estos textos, de los que hay bastantes ejemplos en la red, tienen un particular interés en lo referente a la conciencia lingüística y a la generación de discursos multilingües. El corpus de estudio está compuesto, por tanto, de textos monolingües y multilingües orales y escritos. Los textos han sido numerados correlativamente dentro de su subgrupo -oral o escrito- y se les ha asignado un código que permite identificarlos como monolingües o multilingües, orales 0 escritos $^{14}$. En el presente estudio solo nos centraremos en las interacciones en intercomprensión, por lo que los discursos monolingües no serán objeto de análisis.

Somos conscientes de que trabajar con textos de producción espontánea de la red tiene limitaciones. En primer lugar, no tenemos ninguna información expresa de la edad y, salvo datos esporádicos que revelan los hablantes, no podemos saber en qué rango de edad están los hablantes. Esto nos impide valorar si hay diferencias generacionales en la producción y aceptación de los discursos multilingües, por ejemplo. Tampoco tenemos siempre información sobre el sexo del hablante, porque en la mayoría de los casos los hablantes aparecen con un alias y salvo que declaradamente lo revelen, den algún dato que lo pueda indicar o haya concordancias gramaticales que lo indique, este dato no se puede obtener. Más opacos son aun los datos sobre el tipo de formación que tienen los hablantes que conforman nuestros discursos, puesto que, a excepción de casos puntuales, como son los textos generados por profesores de lengua, que lo dicen claramente, no disponemos de dicha información. La mayoría de estos textos son ajenos a

14 De este modo, los textos monolingües van precedidos de la clave MON, seguida del número de texto y a continuación la letra $\mathrm{O}$ para indicar si es oral o $\mathrm{E}$ si el texto es escrito; por ejemplo, MON10 (texto 1 monolingüe escrito). Para los textos multilingües se ha adoptado el mismo sistema, pero con la clave MUL, por ejemplo, MUL1E (texto 1 multilingües escrito). 
la ocupación de los hablantes y giran mayoritariamente en torno a aficiones o intereses personales que no dejan ver el grado de formación que tienen. Gardner-Chlores (2009: 81-85) señala en su trabajo sobre el cambio de código que estos elementos, de una u otra forma, influyen en las actitudes positivas o negativas hacia este fenómeno. Tampoco tenemos información sobre las lenguas de los hablantes, ni sobre cuál o cuáles son sus lenguas maternas y las lenguas segundas, de modo que este dato no se puede utilizar tampoco para los análisis. Estas carencias, inevitables por la propia naturaleza del corpus, se ven compensadas por otras ventajas que solo pueden ofrecer recopilaciones de textos producidos de forma espontánea. La naturalidad de las interacciones que encontramos en internet nos aleja de uno de los problemas, que desde hace tiempo se observa en los trabajos, en los que los hablantes tienen que dar información sobre su conocimiento y uso de segundas lenguas, la prevaricación (en el sentido clásico), sea o no intencionada. La libertad con la que se expresan los hablantes, en particular en las interacciones escritas, revela el grado lingüístico de aceptación de estas interacciones y sus marcos de producción más favorables.

\section{Resultados y análisis de los datos}

El primer dato que verdaderamente destaca al tratar los discursos multilingües es, precisamente, su escasez en la red. No solamente se trata de un problema en la búsqueda, sino que, realmente, la mayoría de los textos de internet son monolingües. La producción registrada en internet apenas muestra el plurilingüismo de los hablantes. El monolingüismo como ideología y como práctica no es concordante con la realidad extralingüística (Alisaari et al. 2019) La producción textual oral y escrita está dominada por el monolingüismo, tanto desde la lengua materna como desde una segunda lengua. Los intentos de sensibilización al plurilingüismo en la práctica escrita en didáctica de las lenguas, por ejemplo, son una muestra de la voluntad de cambio (Faneca \& Araújo, 2018) pero todavía claramente minoritarios.

El corpus de estudio es una muestra más del valor que los hablantes dan a los textos monolingües frente a los multilingües, en particular cuando consideran que los textos no solamente están cumpliendo una función informativa, en un sentido básico, sino que además tienen otro valor e intencionalidad. Esta distinción entre un discurso informativo y un discurso que presenta una mayor elaboración, que puede deberse tanto a la estructura ${ }^{15}$ como a la relevancia del contenido e intencionalidad, nos permite establecer

15 Es decir, que responda a una tipología textual específica; como es, por ejemplo, un artículo de divulgación o científico. 
una distinción en nuestro corpus. Los textos multilingües tanto escritos como orales son más sencillos compositivamente que los textos monolingües tanto desde el punto de vista de la estructura como de la informatividad/contenido e intencionalidad ${ }^{16}$.

En este estudio nos vamos a centrar en los textos escritos. Estos textos se suelen insertan en foros o en cadenas de comentarios originados por un texto previo (de un blog, por ejemplo). Esto es informativo en sí porque presupone que los lectores de los foros o los seguidores de un usuario de internet entienden la lengua en la que están escritos los textos, aunque pueden no ser competentes en su escritura, por ejemplo. Si no tuvieran una cierta capacidad de comprensión no serían seguidores de una persona o página y no intervendrían. Estos textos se originan, normalmente, como respuesta a una pregunta real o duda de otro usuario de la red, aunque también pueden ser comentarios a un hecho/objeto. Su finalidad es, por tanto, satisfacer la necesidad informativa manifestada en la red por otro usuario, de forma concisa y en general, breve. El contenido temático de estos textos es variado, pero suele girar en torno a aficiones, compras o informaciones puntuales sobre un tema, como, por ejemplo en MUL10E ${ }^{17}$, cuyo tema es la ornitología "Busco arlequim portugués" 18 .

\section{«MUL10E:}

"REYE: Hola me interesaria adquirir ejemplares de arlequin portugués,al menos dos parejas moña $x$ sin moña, gracias

[...]

astavares: Olá, boa noite. Parabéns aos dois pelo gosto em criar o Canário Arlequim Português. Saudações ornitófilas.

REYE: Olá, bons dias amigo Armindo. ¿tudo bom foi-vos in Piacenza com os arlequims? Espero que para o próximo ano seja o reconhecimento definitivo para esta raça de canarios tão bela. Uma pregunta, ¿não saberás de algum criador cerca de Extremadura (Elvas, Campo Maior...) que disponha de casais para vender? Espero tu respuesta. Muito Obrigado e desculpa mi portunhol.

astavares: Olá Reye, boa tarde. Não te preocupes como o teu português. A língua dos nossos países é bastante compreensivel, eu também não sei escrever espanhol e percebo-o bem. Os criadores mais próximos da tua zona localizam-se em Évora o que é bastante longe (são o Rui Godinho, o Rui Silva e o Dário Oliveira) mas tanto quanto sei neste momento já não têm Arlequins para venda. Saudações ornitófilas"."

(1981).

${ }^{16}$ De acuerdo con el contenido que dan a estos términos Beaugrande y Dressler

17 https://www.canariculturacolor.com/foros/showthread.php?t=82132

18 Las transcripciones de los textos son literales. 
La estructura que adoptan estos textos multilingües es dialógica ya que se trata de interacciones, y presentan dos modalidades: (i) estructuras dialógicas de interacción inactiva y (ii) estructuras dialógicas con interacción activa y abierta, como el caso que acabamos de ver en MUL10E. En este caso, hay una interacción entre los usuarios, los cuales reaccionan a un texto mediante opiniones. Pero la interacción puede no ser productiva o limitarse a interacciones muy restringidas, generalmente de persona a persona, como en MUL7E ${ }^{19}$, sobre un alojamiento de airbnb. En este texto puede verse la falta de productividad, ya que entre las entradas hay lapsos de tiempo muy grandes. La conformación del texto no responde a una interacción entre usuarios, en sentido estricto, sino que es el hecho/objeto sobre el que opinan el que articula estos textos, y es globalmente una interacción y así lo interpretan los lectores.

\section{«MUL7E:}

"Roxana: septiembre de 2018. Es un lugar muy bonito, muy tranquilo perfecto para descansar. Moises es un excelente anfitrión. Nos envió con anticipación la información para llegar y ademas nos dio información de los lugares que podíamos visitar.

Mauricio: agosto de 2016. La experiencia fue fantástica en la casa de Moises. Él fue muy atento antes y durante nuestra estadía. El lugar es perfecto, igual que las fotografías. Si regreso a Campos de Jordao volveré a hospedarme en su casa.

Respuesta de Moises: Muchas gracias por sus palavras generosas! Tuve mucho gusto em compartir buenos momentos e platicar com ustedes! Perdon por mi portunhol ! agosto 2016

Gabriel: junio de 2019. Lugar ótimo para quem quer um espaço mais isolado, longe da loucura da cidade em alta temporada. 0 acesso à casa pode ser complicado para alguns, portanto atente-se aos vídeos que o Moisés encaminhar explicando passo a passo. Próximo ao local tem o Mirante da Ferradura que dá pra... Más información.

Melissa: junio de 2019. Ambiente agradável, incrível hospitalidade. A localização não podia ser melhor, vista de perder o fôlego! Casa muito limpa e funcional. E pra fechar, a Luna conquistou nossos corações. Nota 10" [...]»

Las dos modalidades de interacción que estamos analizando -(i) interacción activa y abierta y (ii) interacción inactiva- al producirse en dos lenguas del mismo origen tipológico, como son el portugués y el español, nos llevan a interacciones de intercomprensión espontánea. Esta última forma de interacción (ii) se puede considerar como intercomprensión si tomamos el punto de vista del lector, si se da la circunstancia de que el lector solamente conozca una de las lenguas y estas estén relacionadas tipológicamente. Si

${ }^{19} \mathrm{https}: / / w w w . a i r b n b . c o m . g t / r o o m s / 11944592$ ?source_impression_id=p3_15 63097609_20bzOil6s\%2BJdCCec 
el lector conoce de forma competente las lenguas que aparecen en el texto multilingüe no estaríamos ante un caso de intercomprensión lectora, sino que estaríamos ante un caso de comprensión en L2 ${ }^{20}$. En el ejemplo de MUL7E, la interacción que se produce entre Mauricio y Moisés es un caso de interacción activa insertada en un texto global de interacción inactiva.

Los textos que siguen a continuación, MUL3E ${ }^{21}$ Y MUL5E ${ }^{22}$, al igual que MUL10E, muestra interacciones espontáneas en intercomprensión entre español y portugués correspondientes al modelo (ii):

\section{«MUL3E:}

"Matrix: minha planta ta cheia de manchas brancas e descobri q sao aranhas roxas... borrifando agua com vinagre resolve? abraço a todos.

eumesmo: aranhas roxas??????

marofado: aranha vermelha? será?

Matrix: é Ekeles Bixinhos Pretos Bem Pequenos Q Ficam Atraz Da Folha Deixando Uma Mancha Branca.....a

mescaludo: Mezcla 2 cigarrillos de tabaco rubio en un vaso de agua, despues de 3 dias lo filtras y pulverizas con el liquido las plantas, el vinagre no es solucion para las arañas rojas. PD: no se escribir portugues lo siento.

Matrix: No compriendi una mierda doq hablou jajajaj, sorry

Verdim: po fala serio os caras tem a manha de vir aqui e responder e nego ainda tira uma onda....nao compreendeu matrix? usa o tradutor, ou vc nunca ouviu falar dele....http://traductor.universia.net. "Mistura 2 cigarros de fumo loiro num copo de água, despues de 3 dias o filtras e pulverizas com o liquido as plantas, o vinagre não é solucion para as aranhas vermelhas PD: não se escrever portugues o sento».

mescaludo: Gracias verdim por la traduccion".»

«MUL5E :

"CAMISETAS PAMPLING: AHORA SI... AUTOPSIA

ricardo.pampling: 03 de Octubre de 2007. Pues depués de un primer intento fallido ya tenemos aquí las camisetas de "Autopsia" de Gprod. Una reedición que nos habiais pedido muchos de vosotros y que esta vez viene, como ya es habitual, en camisetas American Apparel. Así que nada... a jugar a los médicos.

${ }^{20}$ No consideramos que el lector sea bilingüe, aunque podría ser el caso, y entonces la interpretación del texto se haría desde la lengua materna.

21 https://www.cannabiscafe.net/foros/forum/otros-pa\%C3\%83\%C2\%ADsesanother-countries/cultivo-no-brasil/problemas-e-deficiencias/64690-agua-com-vinagreacaba-com-as-aranhas-roxas AUTOPSIA

22 https://www. pampling.com/blog/140-CAMISETAS-PAMPLING-AHORA-SI- 
rafario: 3 de Octubre de 2007, 12:05. aeee amigons!!! Estoy aca en Brasil!! Y ya compre una Autopsia..Creo que ustedes van entregar aca.. Pero no se cuando.. rss Ah... si... perdón por mi portunhol..rssss But, let's go boys!!! Saludos brasileños.!

ricardo.pampling: 3 de Octubre de 2007, 12:05. Hola Rafario, tu camiseta acaba de ser enviada como carta certificada a través del "magnífico" servicio postal español. Ya enviamos una antes a Brasil y llegó sin problemas. Ya nos contarás. Un saludo.

rafario: 3 de Octubre de 2007, 12:05. Hola Amigo Ricardo!!! Muy buenooo!!! Mi t-shirt ya esta a camino!!! Y mi portuñol esta terrible... rsss. Pero, mismo así, me gustaría salúdalos por la atención con nosotros!! Ahora yo voy hacer una propaganda de Pampling acá en Brasil! Gracias!!

rafario: 17 de Octubre de 2007, 12:05. Hola amigo ricardo!! Mi camiseta Ilego ayer!!! Muy bonita!! Y aunque recibí un adhesivo!! Muy bueno!!! Saludos brasileños!!"»

Estos textos presentan similitudes que hemos visto repetidas en otras muestras del corpus. La primera de ellas es el motivo de las interacciones. Como hemos señalado, responden a una petición de ayuda sobre un tema de interés de los usuarios. Esto es habitual en muchos foros monolingües, pero lo que no es tan habitual es que un hablante se aventure a responder en otra lengua y colaborar, por tanto, en una creación discursiva multilingüe de intercomprensión. La pregunta es, por tanto, ¿por qué hay hablantes que se 'atreven' a participar? Al margen de actitudes lingüísticas o circunstancias específicas individuales, imposibles de evaluar sistemáticamente en interacciones espontáneas, hay algunos elementos que permiten avanzar una respuesta ${ }^{23}$. Se puede observar en estos ejemplos la familiaridad que los hablantes muestran hacia la otra lengua y que manifiestan de dos formas: (i) pedir disculpas por no ser suficientemente competentes en la otra lengua; y, (ii) intentar comunicarse mediante el uso de portuñol no estable, es decir, con alternancia de código, mezcla de códigos y discurso fusionado en el sentido de Auer (1998), además de adaptaciones morfológicas a la lengua a la que se quiere aproximar el discurso. Este uso del portuñol revela que los hablantes que recurren a él tienen necesariamente conocimientos de la morfología y el léxico de la otra lengua. La intención de usar un código aproximado y el propio reconocimiento de ese código como tal, en el caso que tratamos el portuñol,

${ }^{23}$ En las interacciones inactivas no se introducen estas modificaciones. La explicación podría estar en que la creación de estos textos multilingües no responde a los mismos mecanismos de creación. En estos casos, la conformación del discurso multilingüe de este tipo supone la concatenación de entradas aisladas o que normalmente no están directamente relacionadas, lo que recuerda, en cierto modo a la manera en la que se conforman los libros de firmas, con entradas independientes. Los hablantes simplemente se expresan en una lengua diferente que pude ser comprendida por el hablante que inicia el discurso o el destinatario del discurso, al margen de las intervenciones de otros hablantes que intervienen en el texto. 
revela una clara conciencia lingüística de los hablantes. Pedir excusas por no utilizar la lengua del interlocutor es una muestra de conciencia, y se utiliza, además, para que el interlocutor acepte la introducción de otro código o la modificación del código del interlocutor, lo que normalmente es aceptado en la interacción, como se ve en todas las muestras presentadas. En el caso de MUL3E, Matrix -de manera poco educada y jocosa-, dice que no ha entendido nada de la intervención en español de mescaludo, y es recriminado por un tercer hablante (Verdim), quien considera adecuada la intervención por el fácil acceso a una traducción a través de internet, lo que facilitaría como solución la comprensión. El uso de esta disculpa puede interpretarse también como una forma de la figura de la retórica clásica captatio benevolentiae, es una marca de introducción de cambio de código o, directamente, de la introducción de un código aproximado en los discursos multilingües de intercomprensión espontánea español-portugués.

Algunas de las interacciones de intercomprensión que analizamos muestran, además, una sorprendente conciencia metalingüística por parte de los hablantes en relación a lo que es la intercomprensión, aunque por supuesto sin llegar a utilizar una terminología lingüística. Lo hemos visto en MUL10E: "REYE: [...] Muito Obrigado e desculpa mi portunhol. astavares: Olá Reye, boa tarde. Não te preocupes como o teu português. A língua dos nossos países é bastante compreensivel, eu também não sei escrever espanhol e percebo-o bem. [...]. Por supuesto, las reflexiones lingüísticas en torno al portuñol, entendido como el producto de interacciones portugués-español se muestran con mucha mayor claridad en los textos monolingües de nuestro corpus. No obstante, muchos de estos textos proceden de páginas de enseñanza de lenguas, de profesores de lengua o investigadores; en definitiva, textos no solo metalingüísticos, sino con finalidad metalingüística, lo que no deja de ser, en cierto modo, una distorsión de nuestro objetivo, las interacciones espontáneas en intercomprensión.

\section{Conclusiones}

Las posibilidades comunicativas que ofrecen las interacciones espontáneas en intercomprensión revelan el potencial comunicativo de los discursos multilingües. Pero estos discursos no cuentan, de momento, con una valoración positiva socialmente reconocida, ni siquiera por parte de los hablantes de forma individual. Las situaciones discursivas entre hablantes nativos y no nativos tienden a resolverse mediante el uso de una segunda lengua. Una baja competencia en la L2 hace que los hablantes consideren inviable o fallida la comunicación. El recurso a otras posibilidades comunicativas, como es la intercomprensión, no siempre resulta evidente para ellos. La 
falta de una conciencia lingüística sobre otras posibilidades de comunicación que no pasen por el uso de la L2, como es la intercomprensión, impide a los hablantes desplegar los conocimientos lingüísticos que poseen. Aceptar el discurso multilingüe (y sus distintas modalidades) como un acto comunicativo normal requiere, por tanto, un cambio en la conciencia lingüística de los hablantes. En la medida en la que la posibilidad de establecer interacciones comunicativas en intercomprensión, en tanto que un discurso multilingüe, no sea percibido por los hablantes como una posibilidad discursiva válida, la intercomprensión seguirá estando infrautilizada y restringida a situaciones discursivas esporádicas. El miedo que presentan los hablantes a generar discursos multilingües en los que, además, se dé entrada a variantes inestables de una lengua, como es el caso del portuñol que hemos analizado, necesita ser superado mediante un cambio de mentalidad en relación a la ideología monolingüe. La conciencia lingüística individual es clave para avanzar en la aceptación de las posibilidades comunicativas que ofrece la generación de discursos multilingües.

\section{Referencias bibliográficas}

Alisaari, J., Heikkola, L., Commins, N., \& Acquah, E. (2019). Monolingual ideologies confronting multilingual realities. finnish teachers' beliefs about linguistic diversity. Teaching and Teacher Education, 80, 48-58.

Alvanoudi, A. (2018). Language contact, borrowing and code switching: A case study of australian greek. Journal of Greek Linguistics, 18(1), 3-44.

Álvarez, X.A. (2017). Intangible cultural heritage of the Portugal-Spain border: a contribution from language documentation, MEMORIAMEDIA. Revista do e-Museu do Património Cultural Imaterial, 1. Disponible en https://bit.ly/2JJWbNu

Auer, P. (1998).Code-switching in conversation: Language, interaction and identity. London: Routledge.

Beacco, J.C., Byram, M., Cavalli, M., Coste, D., Cuenat, M. E., Goullier, F., \& Panthier, J. (2010). Guide pour le développement et la mise en œuvre de curriculums pour une éducation plurilingue et interculturelle. Strasbourg: Conseil de l'Europe.

Beacco, J.C., Fleming, M., Goullier, F., Thürmann, E., \& Vollmer, H. (2015). Les dimensions linguistiques de toutes les matières scolaires. Un Guide pour l'élaboration des curriculums et pour la formation des enseignants. Strasbourg: Conseil de l'Europe.

Beaugrande, S., \& Dressler, W. (1981). Introducción a la lingüística del texto (1a. ed.). Barcelona: Ariel, 1997.

Beebe, L.M., \& Giles, H. (1984). Speech-accommodation theories: a discussion in terms of second-language acquisition. International Journal of the Sociology of Language, $46,5-32$. 
Bialystok, E. (2001). Bilingualism in development : language, literacy, and cognition. Cambridge, U.K; New York: Cambridge University Press.

Billiez, J., Degache, C., \& Simon, D.L. (2013). Louise Dabène: un itinéraire hors du commun, Lidil, Revue de linguistique et de didactique des langues, 47, 5-13. Disponible en https://journals.openedition.org/lidil/3259

Campos, G. (2008). Atitutes em fronteira: o caso de Tabatinga e Letícia. Forma y Función, 21, 303-324.

Candelier, M. (coord.) (2008). MAREP. Marco de Referencia para los Enfoques Plurales de las Lenguas y de las Culturas. Graz: CELM. Disponible en https://bit.ly/2SzOVbA

Candelier, M., Camilleri-Grima, A., Castellotti, V., de Pietro, J.-F., Lorincz, I., Meissner, F.-J., Schroder-Sura, A., \& Noguerol, A. (2007). Cadre de Référence pour les Approches Plurielles des langues et des cultures (CARAP). Strasbourg: Centre Européen pour les Langues Vivantes / Conseil de l'Europe.

Carrell, P. (1989). Metacognitive Awareness and Second Language Reading. The Modern Language Journal, 73(2), 121-134.

Castro, S. (2015). Las plataformas galanet y galapro: enseñanza y formación en intercomprensión. In M. Matesanz del Barrio, La enseñanza de la intercomprensión a distancia, 305324. Madrid: Universidad Complutense.

Cheng, T. (2013). Codeswitching and participant orientations in a chinese as a foreign language classroom. The Modern Language Journal,97(4), 869-886.

Corbella, D. \& Fajardo, A. (eds.) (2017). Español y portugués en contacto. Préstamos léxicos e interferencias, Berlin: De Gruyter Mouton.

Coste, D., Moore, D., \& Zarate, G. (2009). Competence plurilingue et pluriculturelle. Vers un Cadre Européen Commun de référence pour l'enseignement et l'apprentissage des langues vivantes: études préparatoires. Strasbourg: Conseil de l'Europe. Disponible en https://bit.ly/2GqOt97

Dewaele, J-M. (1998). Lexical Inventions: French Interlanguage as L2 versus L3. Applied Linguistics 19(4), 471-490

Dabène, Louise (1975). L'enseignement de l'espagnol aux francophones (Pour une didactique des langues « voisines»), Langages, 39, 1-64.

Degache, C., \& Melo, S. (2008). Un concept aux multiples facettes, Les Langues Modernes, Dossier: L'Intercompréhension, 1, 7-14.

Denham, K., \& Lobeck, A. (2010). Linguistics at school: Language awareness in primary and secondary education. Cambridge, UK: Cambridge University Press.

Dewaele, J. (1998). Lexical inventions: French interlanguage as L2 versus L3. Applied Linguistics, 19(4), 471.

Dewaele, J. M. (1998) Speech rate variation in 2 oral styles of advanced French interlanguage. In: Regan, V. (ed.) Contemporary Approaches to Second Language Acquisition in Social Context: Crosslinguistic Perspectives. Dublin: University College Dublin Press, pp. 113-123.

Dewaele, J. M. (2001). Activation or inhibition? The interaction of L1, L2 and L3 on the language mode continuum. In Cenoz, J., Hufeisen, B., \& Jessner, U. (2001). Cross-linguistic 
influence in third language acquisition: Psycholinguistic perspectives (Bilingual education and bilingualism, 31), 69-89. Clevedon, UK: Multilingual Matters.

Di Gesù, F. (2017). Lingüística-didáctica del español para italianos: una sinergia entre intercomprensión y contrastividad. In Martínez-Paricio, Violeta \& Manuel PruñonosaTomás (eds.), Intercomprensión románica, 106-122. LynX A Monographic Series in Linguistics and World Perception - Annexa 23. València: Universitat de València.

DGLDFL (2016). Références: Intercompréhension. Délégation Générale à la Langue Française et aux Langues de France.

Doyé, P., (2005): Intercompréhension. Étude de référence, division des politiques linguistiques. Strasbourg: Conseil de l'Europe.

Elizaincín, A. (1992). Dialectos en contacto. Español y portugués en España y América. Montevideo: Arca.

Elizaincín, A. (2004). Las fronteras del español con el portugués en América. Revista Internacional de Lingüística Iberoamericana: RILI 2, 105-18.

Escudé, P., \& Janin, P. (2010). Le point sur l'intercompréhension, clé du plurilinguisme. Paris: CLE International.

Ellis, R. (2002). Does form-focused instruction affect the acquisition of implicit knowledge? A review of the research. Studies in Second Language Aquisition, 24, 223-236.

Ellis, R. (2004). The definition and measurement of explicit knowledge. Language Learning, $54,227-275$.

Ellis, R. (2005). Measuring implicit and explicit knowledge of a second language .A Psychometric Study. Studies in Second Language Acquisition, 27, 141-172.

Eurobarómetro 386 = Special Eurobarometer 386, 2012. European and their languages.

Etxebarria, M. (1995): El bilingüismo en el estado español. Bilbao: FBV.

Faneca, M.R. \& Araújo, M.H. (2018). Kamishibai plurilingue: um recurso para abordar a diversidade linguística e cultural nas escolas portuguesas. Conference: International Seminar "Innovative academic practices in higher education: How to make them more sustainable. IAPHE'18". Septiembre 2018, Universidad de Aveiro.

Gardner-Chloros, P. (2009). Code-switching. Leiden: Cambridge University Press.

Gardner-Chloros, P., McEntee-Atalianis, L., \& Finnis, K. (2005). Language attitudes and use in a transplanted setting: Greek Cypriots in London. International Journal of Multilingualism 2(1), 52-80.

Garret, P., \& James, C. (1992). Language awareness in the classroom ([1st. ed.] ed., Applied linguistics and language study). London: Longman.

Giles, H. (ed) (2016). Communication Accommodation Theory. Negotiating Personal Relationships and Social Identities Across Contexts. Cambridge: Cambridge University Press.

Karmiloff-Smith, A. (1979). Micro- and macro-developmental changes in language acquisition and other representation systems, Cognitive Science, 3, 91-118.

Hawkins, E. (1984). Awareness of language. An introduction. Cambridge: Cambridge University. 
Hidalgo, R. (2015). De la intercomprensión a la práctica del plurilingüismo una nueva relación entre las lenguas. In M. Matesanz del Barrio, La enseñanza de la intercomprensión a distancia, 175- 195. Madrid: Universidad Complutense

Horwitz, K. (1988). The beliefs about language learning of beginning university foreign language students. The Modern Language Journal, 72, 283-294.

Horwitz, K. (1999). Cultural and situational influences on foreign language learners' beliefs about language learning: A review of BALLI studies, System, 27, 557-576.

James, C. (1999). Language awareness: implications for the language curriculum, Language, Culture and Curriculum, 12(1), 94-115.

Jee, M. J. (2017). Examining beliefs about language learning in relation to perceived linguistic self-confidence: Learners of Korean as a foreign language in Australia. Linguistic Research, 34(Special Edition), 25-48.

Jessner, U. (2005). Multilingual metalanguage, or the way multilinguals talk about their languages. Language awareness, 14(1), 56-68.

Jessner, U. (2006). Linguistic awareness in multilinguals: English as a third language. Edinburgh: Edinburgh University Press.

Kennedy, S. (2012). Exploring the relationship between language awareness and second language use.Tesol Quarterly, 46(2), 398-408.

Leite de Vasconcelos J. (1955). Filologia barranquenha: Apontamentos para o seu estudo. Lisboa: Imprensa Nacional.

Lipski, J.M. (2006). Too close for comfort? The genesis of "Portuñol/Portunhol”. In T. L. Face \& C. A., Klee Selected proceedings of the 8th Hispanic Linguistic Symposium. Cascadilla Proceedings Project, 1-22.

Lipski, J.M. (2009). Searching for the origins of Uruguayan Fronterizo dialects: radical codemixing as "fluent dysfluency". Journal of Portuguese Linguistics, 8(1), 3-44.

Lipski, J.M. (2017). Portuguese/Portuñol in Misiones, Argentina: Another "Fronterizo"? In. M. González-Rivera \& S. Sessarego (Eds.). (2015). New perspectives on hispanic contact: Linguistics in the americas (Lengua y sociedad en el mundo hispánico, 35, 253-281. Madrid: Iberoamericana.

Locane, J.J. (2015). Disquisiciones en torno al "portunhol selvagem". Del horror de los profesores a una "lengua pura". Perífrasis, 6(12), 36-48.

Matesanz del Barrio, M. (2015). Límites discursivos de la intercomprensión en entornos profesionales. En M. Matesanz del Barrio (ed.). La enseñanza de la intercomprensión a distancia (pp. 163-181). Madrid: Universidad Complutense. Disponible en http:// eprints.sim.ucm.es/35033/

Matesanz del Barrio, M. (2017). La intercomprensión tipológica en el enclave de las lenguas de los europeos. En V. Martínez-Paricio y M. (coords.) Intercomprensión románica (pp. 123-140). Valencia: Universidad de Valencia.

Matesanz del Barrio, M. (2019). Discours multilingue et intercompréhension. Université Paul Valéry. Montpellier 3.Séminaire DIPRALANG. 11 mars 2019 (conferencia).

Moreno Cabrera, J. (2016).Multilinguïsmo y lenguas en contacto (Claves de la lingüística). Madrid: Síntesis. 
Moreno, F. (2001). El español en Brasil: logros, dificultades y falsas creencias. Revista de Occidente, 240, 82-99.

Navas, M. (1998). La frontera lingüística hispano-portuguesa. Estado de la cuestión. Madrygal: Revista de estudios gallegos, 1, 83-90.

Navas, M. (2011).El barranqueño : Un modelo de lenguas en contacto ( $1^{\text {a }}$ ed., Línea). Madrid: Universidad Complutense.

Navas, M. (2014). Presente, pasado y futuro de una lengua de contacto lusoespañola. In Fronteras y diálogos. El español y otras lenguas, J. M. Santos Rovira (ed.), 89-100. Lugo: Axac.

Navas, M. (2015). Actitudes lingüísticas en una situación de contacto de lenguas. Revista de Filología Románica, 32(1), 11-20.

Pinto, M. (1999). La consapevolezza metalinguistica: Teoria, sviluppo, strumenti di misurazione (Psicologia del bilinguismo, 1). Pisa: Istituti editoriali e poligrafici internazionali.

Pinto M. A. (2015). La Consapevolezza metalinguistica: Concetti e strumenti di misurazione in lingua italiana e tedesca. Venerdi 4 Settembre 2015. Schweizer Schule Roma. Via Savoia. Roma.

Pires, M.E. (2017). "Portunhol selvagem": Translinguagens em cenário translíngue/transcultural de fronteira. Gragoatá, 22(42), 523-539.

Real Academia Española (2014). Diccionario de la lengua española (23. a ed.). Madrid, Espasa Calpe.

Riley, P. (1997). The guru and the conjurer: Aspects of counselling for self-access. In P. Benson \& P. Voller (eds.), Autonomy and Independence in Language Learning, 114-131. London: Longman.

Rojas, S.L. (2008). Aproximación al estudio de las actitudes lingüísticas en un contexto de contacto de español y portugués en el área urbana trifronteriza Brasil- ColombiaPerú. Forma y Función, 2, 251-285.

Roubaud, M.R. (2010). Les publications de Claire Blanche-Benveniste. https://bit.ly/2SAznCF.

Spiță, D., Lupu, M., Nica, D., \& Nica, I. (eds.) (2016). Les approches plurielles dans l'éducation aux langues: L'intercompréhension, en présence et en ligne. Iaşi: Editura Universităţii, Alexandru loan Cuza

Sukwon, N. \& Yang-soo, J. (2015). A Study on the Korean University Students' Use of Code Switching in Interactional Conversation. Modern Studies in English Language and Literature 59(2), 73-96.

Street, R.J. \& Giles, H. (1982). Speech accommodation theory: a social and cognitive approach to language and speech behavior. In M. Roloff, C. Berger (eds.). Social cognition and communication, Beverly Hills: Sage.

Sturza, E.R. (2004). Fronteiras e práticas lingüísticas: um olhar sobre o portunhol. Revista Internacional de Lingüística Iberoamericana. RILI, I (3), 151-160.

Sturza, E.R. (2005). Línguas de fronteira: o desconhecido território das práticas lingüísticas nas fronteiras brasileiras. Ciência e Cultura, 57(2), 47-50.

Sturza, E.R., \& Tatsch, J. (2016). A fronteira e as línguas em contato: uma perspectiva de abordagem Cadernos de letras de UFF Dossiê: Línguas e culturas em contato, 26(53), 83-98. 
Torres-Cacoullos, R., \& Travis, C. (2016). Two languages, one effect: Structural priming in spontaneous code-switching. Bilingualism: Language and Cognition, 19(4), 733-753.

Waltermire, M. (2012). The differential use of Spanish and Portuguese along the UruguayanBrazilian border. International Journal of Bilingual Education and Bilingualism, 15(5), 509-53.

Weinreich, Uriel. [1953] 2011. Languages in Contact: French, German and Romansch in language contact, borrowing and code switching in Twentieth-Century Switzerland. With an Introduction and Notes by Ronald I. Kim and William Labov. Amsterdam: John Benjamins. 